\title{
Interfacing Aspects Between the Picture Archiving Communications Systems, Radiology Information Systems, and Hospital Information Systems
}

\author{
Claudine M. Breant, Ricky K. Taira, and H.K. Huang
}

\begin{abstract}
Information relevant to radiological applications is commonly managed by several autonomous medical information systems including hospital information systems (HIS), radiological information systems (RIS), and picture archiving and communications systems (PACS). In this report, we explain the need to coordinate these systems and to provide some framework in which they can exchange information. In the first half of this report, we describe the integration of a PACS system into a hospital operation. Next, we present the interfacing methods between the HIS and the RIS, and between the RIS and the PACS. Two methods are further detailed for the communication between the RIS and the PACS (1) the triggered database to database transfer, and (2) the query protocol. The implementation of the first method successfully allows RIS reports, procedure and patient demographic information to be displayed at the request of the user along with the associated images at a PACS workstation. The query protocol allows a PACS to dynamically query RIS information. It will be eventually integrated into the design of a scientific multimedia distributed medical database system built on top of the HIS, the RIS, and the PACS.
\end{abstract}

Copyright $\mathbb{C} 1993$ by W.B. Saunders Company

KEY WORDS: PACS, HIS-RIS-PACS interfacing, HIS, RIS, Medical Information Systems.

$\mathbf{V}$ ARIOUS information systems in hospital environments independently address the management of certain subsets of medical and administrative information using conventional database management methods. Radiological picture archiving and communication systems (PACS) allow image data from once autonomous imaging systems to be sent to general purpose networked computers and provide efficient communication, retrieval, and display of radiological images. Radiological information

From the Medical Imaging Division, Department of Radiological Sciences, UCLA, Los Angeles, CA 90024-1721.

Supported in part by Public Health Senice grant no P01CA51198 and ROICA39063 awarded by the National Cancer Institute, Department of Health and Human Senices.

Address reprint requests to Claudine $M$. Breant, $P h D$, Medical Imaging Division, Department of Radiological Sciences, UCLA, Los Angeles, CA 90024-1721.

Copyright 1993 by W.B. Saunders Company

0897-1889/93/0601-0006\$05.00/0 systems (RIS) manage patient scheduling, requisition information (examination type, preparation required, and reason for examination), imaging technique information, and text interpretations of studies. Hospital information systems (HIS) manage patient demographics, insurance information, clinical scheduling, laboratory reports, patient history, and patient examination information. Digital dictation systems store and playback dictated voice reports from radiology departments. These information systems operate autonomously and have their own history and evolution. Lately, it has become increasingly important to consider some framework to unify the coordination of HIS, RIS, and PACS, to consider their interrelationships, and to allow these systems to exchange information.

For radiological applications, there is currently no framework to integrate and to process multimedia data stored in several distributed medical information systems. This clearly limits the querying power for the radiology department's clinical usage which is concerned in particular with correlating radiographic features stored in PACS, HIS, and RIS. The diagnostic process requires the search for corresponding radiological images in the PACS, the retrieval of patient and examination information from the RIS and the HIS, and the gathering of the results for further processing (ie, determining the diagnosis of the patient). PACS, RIS, and HIS are likely to form a set of heterogeneous database systems, in which each database may use a different query language, data model, and access technique. Therefore, retrieving the data to answer high level clinical and research questions is no simple matter.

\section{INFORMATION SYSTEM ACCESS DESIGN}

Recent advances in network communication technology make it feasible to efficiently and reliably interconnect different computers. The access to an external information system can be designed in three different ways with varying degrees of complexity as described below. 


\section{Terminal Emulation}

This method allows a system to emulate the terminal of a remote host computer and allows the user to perform any of its functions. For example, the user at a PACS workstation can emulate the RIS terminal and can view the diagnostic reports for a specific patient. The user can also perform RIS administration functions including scheduling a new examination, updating patient demographics, or recording a film movement. However, this method does not provide data exchange nor system integration. The user is therefore required to master different database systems and interfaces. In addition, RIS and HIS cannot emulate the PACS terminal because the configuration of PACS is too specific.

\section{Database to Database Transfer}

A database to database transfer allows two or more information systems to share a subset of information by storing in their local databases data from a remote system provided by a communications network. This operation includes making the external data that are meaningful to the local information systems. Some software is necessary to reformat this data according to the local conventions for the data representation before data storage.

\section{Heterogeneous Distributed Database System}

A heterogeneous distributed database system (HDDS) provides a single interface and language to access data distributed in several heterogeneous database systems and allows the data to appear as if it were managed by one single integrated database. A query protocol is responsible for analyzing the requested information, identifying the databases required to answer it, fetching the information, assembling the results, and presenting the results to the user. Ideally, all this is done transparently and without affecting the autonomy of each database system. This process is not easy to achieve because currently available commercial database management products do not provide the means to share information or to communicate with other database management systems types. ${ }^{1}$ Much recent work has been devoted to this area by the computer science community which is interested in finding theoretical approaches to solve the problem. ${ }^{2-4}$

In the first half of this report, we describe the integration of a PACS system into the hospital operations. The exchange of information between the PACS and the two existing information systems, HIS and RIS, is required for a better acceptance of the PACS within both the clinical and the administrative environment. The interrelationships between the HIS, RIS, and PACS are defined. In the second half of the report, we use the interface implementation between the HIS, RIS, and PACS in our medical center as an example to illustrate the concept. 5 Other related experiences in integrating or interfacing medical information systems are described in. ${ }^{6-9}$

\section{PROBLEM DEFINITION IN RADIOLOGY}

\section{HIS, RIS, and PACS}

The issues, design, and goals of various medical information systems including HIS, RIS, and PACS have been described in many ways. ${ }^{7,10,11}$ Traditionally, the HIS was the first computerized management system to appear in the hospital environment, and it handles a large variety of tasks within which three categories can be distinguished: (1) the support of clinical and medical patient care activities in the hospital, (2) the administration of nonpatient care activities of the hospital (financial, personnel, payroll, bed census, etc), and (3) the management and control operations assisting in long-range planning, evaluation of hospital performance, and costs.

The RIS was probably developed independently because of the limited support offered by the HIS to handle information related to the radiology department. The RIS is designed to support both the administrative and the medical part of a radiological department, to reduce administrative overhead, and to improve the quality of medical work. Therefore, the RIS manages mostly general patient demographics and billing information, procedure descriptions and scheduling, diagnostic reports, patient arrival scheduling, film location, film movement, and room scheduling. The RIS configuration is very similar to the HIS, except the RIS is of a smaller scale. The RIS equipment consists of a departmental computer with peripherical de- 
vices such as alphanumeric terminals, printers, and bar code readers attached to it. At present, RIS implementations are autonomous systems or have limited access to HIS. However, some HIS offer embedded RIS subsystems with a high degree of integration.

PACS arose from the radiologist's need for the availability of digital images in a timely fashion and from the referring physician's need for images and related radiology consultation at any time of the day. PACS must also efficiently store and retrieve historical images. Finally, it must provide the radiologist with tools to enhance the viewing and processing of multimodality images.

\section{Interfacing PACS with HIS and RIS}

In a hospital environment, interfacing the PACS, RIS, and HIS has become necessary to enhance the following functions.

Diagnostic process. The diagnostic process at the PACS display station not only includes the recall of images that show the case of interest but also the display of various textual information describing the patient history and previous studies. ${ }^{12}$ Along with the image data and the image description, a PACS should also provide all related text information acquired and managed by the RIS and the HIS in a way that it can be useful to the radiologist during the diagnostic process. RIS and HIS information such as clinical diagnosis, radiological reports, and patient history is indeed necessary at the PACS workstation to complement the images of the cases being viewed.

PACS image management. Some information provided by the RIS can be integrated into PACS image management algorithms to optimize the routing of the image data on the network to the requesting locations. In the PACS database, huge volumes of data are stored (in the magnitude of terabytes). To handle such volume of data, some PACS designers $^{11,13}$ have opted for a system architecture supporting two layers of storage. The first is the central archive system (relatively slow but having a huge storage capacity) where all the images are permanently archived. The second is the local archive system at the workstation (fast but with limited capacity) that contains images for immediate access. Such a storage method needs additional HIS and RIS information to bring images to the workstation before they are requested by the user. The design of a prefetch algorithm for images is influenced by several parameters such as patient status (admitted, discharged, transferred), requisition information, and patient location; all of which are normally stored in the RIS and the HIS.

RIS administration. The planning of a digitalbased radiology department requires the reorganization of some administrative operations performed by the RIS. PACS need to share some RIS information such as the image archive status and the image data file. RIS administration operations would also benefit from the HIS by acquiring some knowledge about patient admission, transfer, and discharge.

Research and teaching. Most of the research and teaching in radiology involves mass screening of clinical cases to determine what constitutes a normal versus an abnormal patient condition for a given patient population. The corresponding knowledge includes diverse types of information that must be correlated, such as image data, results from analyzed images, medical diagnosis, patient demographics, study description, and various patient conditions. At the PACS research workstation, mechanisms must be provided to access and to retrieve data from either the HIS or the RIS when looking for detailed medical and patient information related to particular image data. The cooperation between diverse medical database systems such as HIS, RIS, and PACS is therefore critical to successfully address research and teaching issues in radiology.

\section{ISSUES AND SOLUTIONS FOR INTERFACING} HIS, RIS, AND PACS AT THE UCLA MEDICAL CENTER

\section{Methods and Materials}

For historical reasons, the UCLA Medical Center PACS, RIS, and HIS were designed independently. Currently, they can operate autonomously. In this section, we describe the configuration of these three information systems in our institution as well as the problems to be solved to realize their interconnection. Interfacing methods that have been implemented and future plans are detailed.

\section{The PACS, RIS, and HIS at UCLA}

The process of accessing, integrating, and interpreting the medical data distributed among the heterogeneous 
databases in the HIS, RIS, and PACS is complicated by many factors. The HIS, RIS, and PACS at UCLA are based on different data models and various query languages. It is difficult to have a unified view of data because of differences in naming of data, scaling conventions, and interpretation of data. Finally, the heterogeneous database systems are implemented on different hardware and operating systems.

The PACS. The UCLA PACS architecture and functionalities have been described elsewhere. ${ }^{11.13 .14}$ Images and alphanumeric data are managed by a centralized database management system including: (1) an image archive system composed of an archive server (SUN SPARC server 490 , SUN Microsystems, Mountain View, CA), with an optical library (KODAK ADL 6800, Eastman Kodak Co, Rochester, NY), and (2) a relational database server Sybase (Emeryville, CA) running on a SUN (SUN SPARC server 490). The structured query -language (SQL) is the data manipulation language used to retrieve or insert data. ${ }^{15}$

Information is displayed on high resolution PACS display stations placed in various sections of the radiology department. Each workstation is composed of a SUN 4/470 or 4/370 host computer, a high-speed Concept-51 disk array (Storage-Concept Inc, Irvine, CA), and two 2,048 $\times 2,560-$ pixel display monitors (AVP, Mega Scan Technology Inc, Hopkinton, MA).

The RIS. The RIS is supported by a VAX 6200 computer under Virtual Memory System. The RIS software consists of a MAXIFILE (DMI Co, Minneapolis, MN) user interface to a database system using MUMPS (Massachusetts General Hospital Utility Multiprogramming System). MUMPS is a multiuser database management system that consists of an interactive, high level programming language and a data management facility. ${ }^{16}$

The HIS. The HIS is supported by an IBM 3090/400J (IBM, Purchase, NY) mainframe computer under Multiple Virtual Storage running the Information Management System database facility. Its mission is to provide automated systems to support clinical operations and business operations, medical professionals, executives decisions, and improve medical care. The medical center computing services (MCCS) provide some links between the HIS and various hospital subsystems including the RIS through the external systems communication interface (ESCI) as shown by Fig 1.

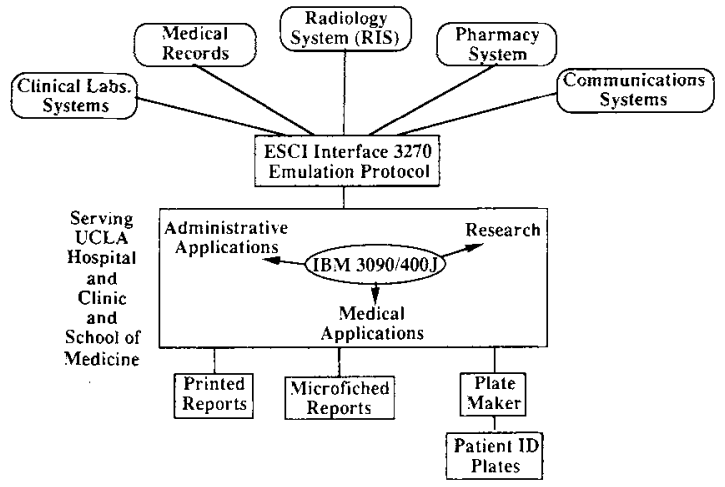

Fig 1. The configuration of the medical center computing services (MCCS) of the HIS at UCLA.
The ESCI facilitates the communication of data between the IBM host mainframe and a remote machine. The communication is through a standard 3270 emulation proto$\mathrm{col}$ in which the external system appears as a terminal for the IBM mainframe. The data traffic is therefore controlled through standard control commands.

Figure 2 shows the ideal configuration in which the three heterogeneous medical information systems at UCLA are interfaced to one other. In this figure, the information exchanges among the HIS, the RIS, and the PACS are bidirectional. Figure 3 presents the existing links and the types of information that each system (HIS, RIS, and PACS) declares to be available at a global level. In our implementation, HIS data is currently available to the PACS through the RIS. For this reason, our interface is not the ideal interface as shown in Fig 2. Instead, it is an interface between the HIS and the RIS, and the RIS and the PACS, shown in Fig 3. Medical information from the PACS is not transferred back to the RIS, nor does the HIS accept queries and acknowledgments.

\section{Assumptions}

Our interfacing methods assume the following. (1) Each system (HIS, RIS and PACS) remains unchanged in its configuration, data, and functions performed. (2) Each system is hardware and software extended to allow the communication with other systems. (3) Only data is shared, functions remains local. For example, RIS functions can not be performed at the PACS or the HIS workstation, keeping the specificity and autonomy of each system and simplifying our interface because database updates will not be allowed on a global level.

Based on these assumptions, successfully interfacing different information systems includes the following tasks: (1) Identify and uniformly represent for each system the subset of data that it agrees to share with the other systems. Access rights and authorization problems are solved during this process. (2) Make the shared data meaningful. This task consists of designing a high level presentation, solving data inconsistencies, and naming conventions. This can be done by using a common data model and data language, and by defining rules of correspondence between various data definitions. (3) Define the protocol of data transfer, and define some standardization methods.

\section{Database to Database Transfer Method}

Figure 3 represents the database to database transfers of information between the HIS and the RIS and between the RIS and the PACS in our department. They are supported by the ESCI interface module between the HIS and the RIS computers and by an Ethernet network link between the RIS and the PACS database machines. Figure 3 also shows the types of information that are being shared. We use the patient identification number, which is the same for all three information systems, to correlate remote and local data.

The HIS-RIS and the RIS-PACS interfaces consist of two levels: the application level and the communication level. The application level consists of the management of the events that trigger the transfer of data from one information 


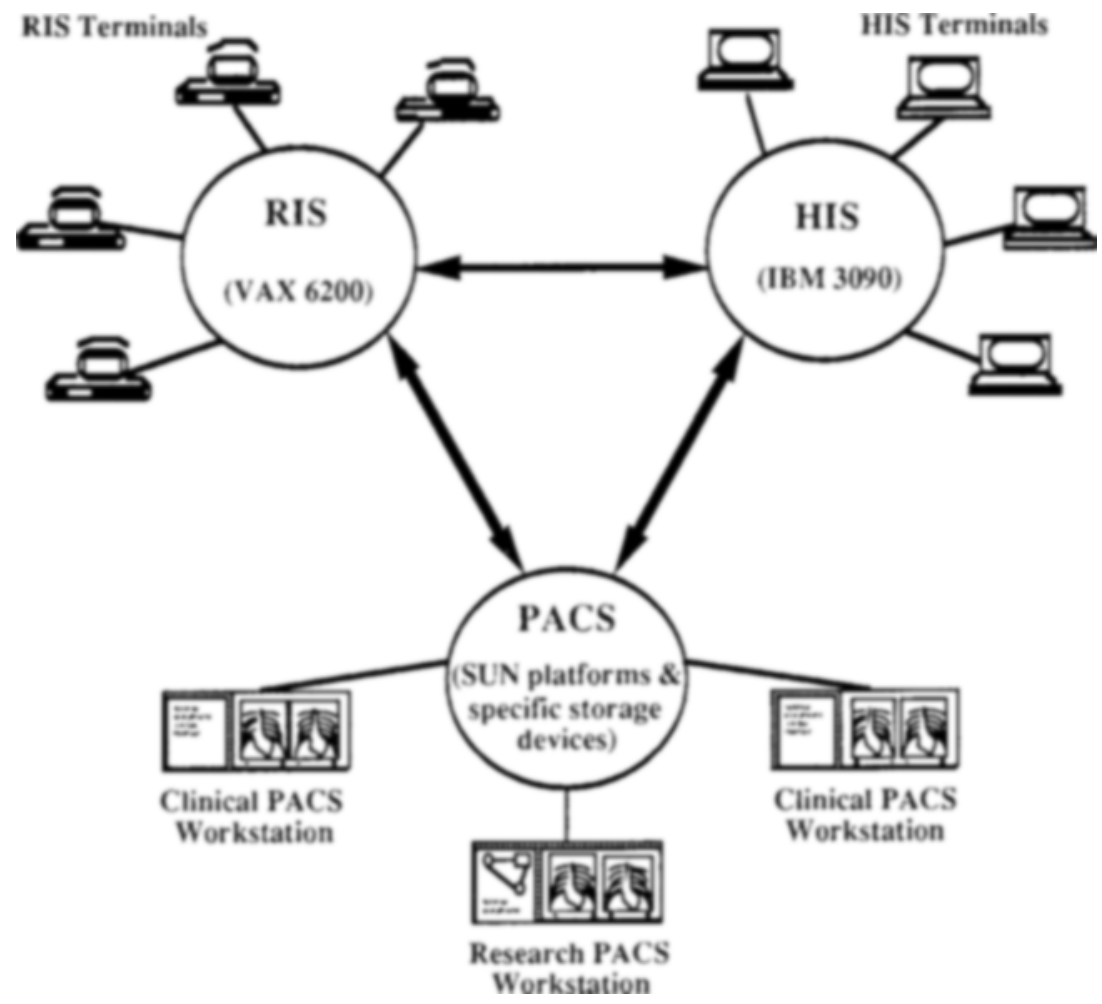

Fig 2. An ideal total medical information system in which interconnections between the HIS, RIS, and PACS are direct and bidirectional. system to another one. The significant HIS, RIS, and PACS events and the associated database actions triggered by each system are depicted in Fig 4. The communication level manages the flow of data between the different computers.

HIS to RIS. Information about patient admission, transfer, and discharge is integrated into the RIS database to update the patient administrative information. The HIS events of interest to the RIS are the admission, transfer, and discharge of a patient. For each event, the related patient demographic information is sent to the RIS and then stored in its database.

As shown in Fig 1, the communication level between the HIS and the RIS is based on the standard 3270 emulation protocol provided by the ESCI module. In this terminal emulation configuration, the HIS mainframe computer treats the RIS as a locally defined 3270 terminal. The data

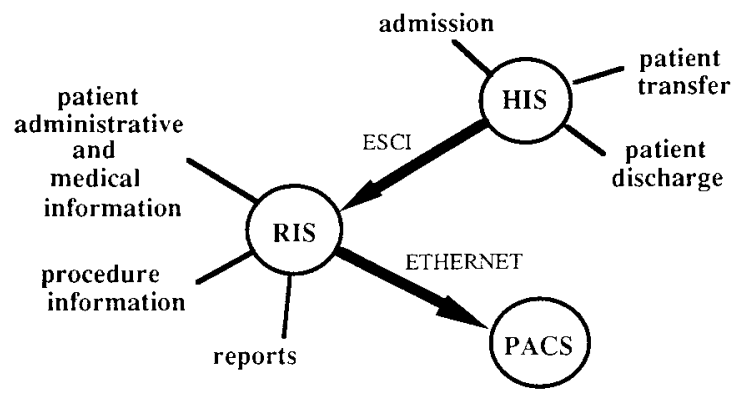

Fig 3. Current architecture of information exchanges between the UCLA PACS, RIS, and HIS. This figure also shows the types of information that are being shared among the three databases. link comprises two RS-232 American Standard Code for Information Exchange lines as asynchronous lines for the communications interfaces. These lines support information messages from the HIS to the RIS as well as acknowledgment messages from the RIS.

RIS to PACS. The following events occurring in the RIS are notified to the PACS: patient arrival, admission, discharge, transfer, procedure complete, and report approval. For example, each time a report is approved, the corresponding text report, the author of the report, the date and time of the approval are automatically stored into a sequential file which is forwarded to the PACS database server machine. Some data such as dates and times are reformatted using the PACS Sybase database conventions. The resulting information is then stored in the PACS relational database.

To implement this RIS-PACS interface, the RIS pro-

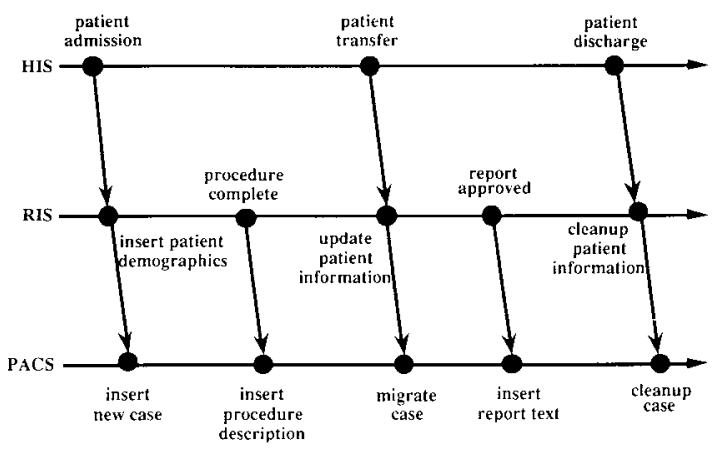

Fig 4. Information transfer from the HIS to the RIS, and from the RIS to the PACS. 


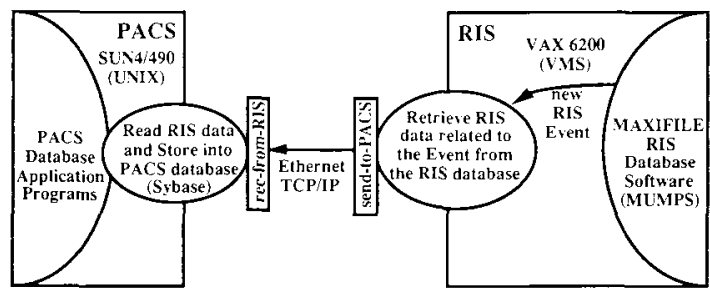

Fig 5. RIS-PACS interface architecture implemented using a database-to-database transfer with a trigger mechanism.

grams have been first extended to record the relevant RIS events. The application level of the interface waits for the occurrence of an event and triggers the corresponding data extraction, data gathering, formatting, and file packaging. The communication level manages the transfer of the file to the PACS database machine using the two processes send-to$P A C S$ and rec-from-RIS. Our effort to standardize the communication includes the use of a standard network protocol transmission control protocol/internet protocol (TCP/IP) to send the data in the ASCII format on the Ethernet network. The PACS machine receives this data and stores it for subsequent use into the PACS Sybase database tables. The architecture of this interface is depicted in Fig 5.

\section{RESULTS}

The interfaces between HIS, RIS, and PACS are currently operational at the UCLA Medical Center. A subset of the HIS and RIS data is available to the PACS. Our first application makes use of these interfaces as part of our goal to design some documentation tools at the UCLA PACS workstation. ${ }^{12}$ In this PACS environment, images are viewed from high resolution 2,048 $\times 2,560$-pixel monitors. During the diagnosis process, different tools are provided to the radiologists to select images, study descriptions, and to perform basic image processing operations. Radiological reports are first recorded by the radiologists and then transcribed at the RIS terminals. On approval, the radiological reports are automatically forwarded to the PACS. Then, radiological text reports, procedure, and demographics information can be displayed at the request of the user along with the associated images at the same PACS workstation. This feature is useful not only to convey a complete diagnosis to the referring physicians but will also be helpful to the radiologist when viewing future studies of a patient.

\section{DISCUSSION}

Interfacing the medical systems (HIS, RIS, and PACS) is necessary for optimizing patient care process in a hospital. In this report, we described the interfacing method using a triggered database to database transfer. This method is optimal when a small amount of predefined remote information needs to be systematically and rapidly available to a local information system. The local storage of information ensures fast access to this data. However, besides requiring additional local disk space, the redundant storage of data brings some difficult management overhead to keep the data consistent among the different databases. This is especially important when the data gets updated. When a huge amount of remote information must be locally available, disk space problems and the requirements for handling data redundancy can prohibit the use of this method. For these reasons, a query protocol method is being implemented for providing dynamic access to remote information systems without the disadvantages of the database to database transfer method.

This query protocol allows one to access data managed by a set of HDDSs. This work is currently under development and is part of our effort to build a distributed multimedia scientific medical database system. ${ }^{17,18}$ This system is an HDDS that allows the user to retrieve textual information as well as image data. It is built on top of the PACS, the RIS, and the HIS. Using a PACS research workstation, the user will be able to retrieve information uniformly from either HIS or RIS system and to automatically integrate the results into one answer. The standardization of this interface includes the following: (1) the use of SQL as the global query language, (2) the use of the relational data model as the global data model, (3) the use of the TCP/IP network communication protocol, and (4) the transfer of data using the ASCII format (Fig 6). At the present time, the commu-

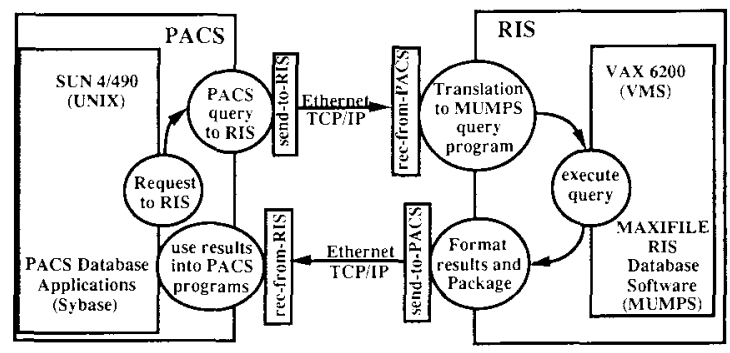

Fig 6. RIS-PACS interface architecture implementing a query protocol. 
nication level of the query protocol is operational between the RIS and the PACS. Using the PACS research station, it is possible to access to RIS data through a set of predefined SQL queries. Thus, it requires no dynamic translation operation into the MUMPS language.

The two methods (query protocol and database to database transfer) can be used together to combine their advantages. For example, in our department, the PACS database stores the RIS diagnosis reports for only the few days that they are needed in a timely fashion. After this period, they are automatically deleted from the PACS database. Later on, any old report can be accessed from RIS at the PACS workstation on the explicit request of the user by using the query protocol method. In our current imple- mentation, one or two seconds are needed to retrieve a report using the database to database transfer method, ie, from the RIS to the PACS. For the same operation, the query protocol method, which includes two data transfers on the network and one remote query processing operation, is 20 times slower. However, the advent of high-speed networks should eventually provide the means for a rapid access to remote data with the sole query protocol approach.

\section{ACKNOWLEDGMENT}

The authors would like to thank Tom Sowle, director of the Information Systems and Medical Center Computing Services at the University of California at Los Angeles (UCLA) Medical Center and Greg Tashima, manager of the RIS at the UCLA Medical Center, for their contributions in providing necessary resources for the interfaces.

\section{REFERENCES}

1. Hsiao DK, Kamel MN: Heterogeneous databases: Proliferations, issues, and solutions. IEEE Trans Knowledge Data Eng 1:45-62, 1989

2. Breitbart Y: Proc Workshop Multidatabase and Semantic Interoperability. workshop report, Department of Computer Science, Lexington, KY, University of Kentucky, 1990

3. Kambayashi Y, Rusinkiewicz M, Sheth A (eds): IMS91: Proc First International Workshop Interoperability Multidatabase Systems. Los Alamitos, CA, IEEE CS, 1991

4. Yu C: Proc NSF Workshop Heterogeneous Database Systems. conference report, Department of Electrical Engineering and Computer Science, Chicago, IL, University of Illinois, 1989

5. Breant CM, Taira RK, Tashima GH, et al: Issues and Solutions for Interfacing a RIS with a PACS database. Proceeding of the SPIE Medical Imaging VI, Newport Beach, 1992

6. Bakker AR, Kouwenberg JML, Ottes FP: HIS and PACS Integration Aspects. Amsterdam, North Holland, Medinfo 89, 1989, pp 377-381

7. Rienhoff O, Greinacher CFC (eds): A general PACSRIS interface, Lecture notes in Medical Informatics. Springer-Verlag, Berlin, 1988

8. Arenson R, Seshari S, Stevens F, et al: The overlapping domains and interface between radiology information system and medical image management system (PACS). Proc CAR, 1987, pp 855-865

9. Heu R, Hass R, Scharnberg B: Implementation of a data exchange procedure between RIS and PACS. Proc SPIE 626:594-596, 1986
10. Bakker AR, Ball MJ, Scherrer JR, et al (eds): Towards New Hospital Information Systems. Amsterdam, North-Holland, 1988

11. Huang HK, Taira RK: PACS Infrastructure Design. AJR Am J Roentgenol 158:743-749, 1992

12. Breant CM, Taira RK, Sinha U, et al: Documentation and playback of a radiological diagnosis in a PACS environment. Radiology 181:283, 1991 (abstr)

13. Taira RK, Mankovich NJ, Boechat MI, et al: Design and implementation of a picture archiving and communication system (PACS) for pediatric radiology. AJR Am J Roentgenol 150:1117-1121, 1988

14. Huang HK, Kangarloo H, Cho PS, et al: Planning a totally digital radiology department. AJR Am J Roentgenol 154:635-639, 1990

15. Stewart BK, Taira RK: Database architecture and design for the PACS, in Huang $\mathrm{HK}$, et al (eds): Picture Archiving and Communication Systems in Medicine. NATO ASI. Series. Berlin, Germany, Springer-Verlag, 1991, pp 83-89

16. Kuzmak PM: The Challenge of MUMPS for the 1990's: Interface MUMPS to Mainstream Computing," MUMPS Users Group Quarterly XIX:1, 1989

17. Chu WW, Ieong IT, Taira RK, et al: A Temporal Evolutionary Object-Oriented Data Model for Medical Image Management. IEEE 5th Symposium on ComputerBased Medical System, Durham, NC, 1992

18. Cardenas AF, Ieong IT, Barker R, et al: The knowledge-based object-oriented PICQUERY + language. IEEE Trans Data Knowledge Eng (submitted) 